\title{
Comparison of Antibacterial Efficacy of Locally Produced Alcohol Based Hand Sanitizer and Commonly Available Commercial Hand Sanitizer Used in Healthcare Facilities in Uganda
}

\author{
Tusabe Fred1,2*, Kasuswa Sophia1, Ssegawa Alex1, Busiinge Emmanuel1, Lusheda Tom1, \\ Ampaire Lucas ${ }^{1}$
}

${ }^{1}$ Mbarara University of Science and Technology, Mbarara, Uganda

${ }^{2}$ Infectious Diseases Institute, Kampala, Uganda

Email: *tusabef@gmail.com

How to cite this paper: Fred, T., Sophia, K., Alex, S., Emmanuel, B., Tom, L. and Lucas, A. (2020) Comparison of Antibacterial Efficacy of Locally Produced Alcohol Based Hand Sanitizer and Commonly Available Commercial Hand Sanitizer Used in Healthcare Facilities in Uganda. Open Access Library Journal, 7: e6221.

https://doi.org/10.4236/oalib.1106221

Received: March 12, 2020

Accepted: April 4, 2020

Published: April 7, 2020

Copyright $\odot 2020$ by author(s) and Open Access Library Inc.

This work is licensed under the Creative Commons Attribution International License (CC BY 4.0).

http://creativecommons.org/licenses/by/4.0/

\begin{abstract}
Use of hand sanitizers has become a cornerstone in clinical practice for the prevention of disease transmission between practitioners and patients. There are a number of hand sanitizers sold on the Ugandan market with labels on their packages that claim that the hand sanitizer can kill $99.999 \%$ of germs and also there are hospitals that embarked on the local production of alcohol based hand sanitizer whose efficacy data are not locally available. Objective: To evaluate antibacterial efficacy of locally produced alcohol based hand sanitizer and commonly available commercial hand sanitizer used in healthcare facilities in Uganda. Method: This was an in vitro experimental, laboratory-based study of two different brands of hand sanitizers commonly used in healthcare facilities in Uganda and these were compared to a reference standard 60\% Isopropyl alcohol. Efficacy was evaluated using standard organisms of Klebsiella pneumoniae American Type Culture Collection (ATCC 13883), Escherichia coli (ATCC 25922), and S. aureus (ATCC 25923) as per prEN12054, a European standard method. The logarithmic and percentage reduction factors (RF) were assessed at baseline and after treatment. Results: Both hand sanitizers studied were able to reduce bacteria by more than $10^{5}$-fold within 15 seconds. Efficacy was independent of the alcohol concentration in each brand (Saraya 70\% and locally made $80 \%$ alcohol). Conclusion: All of the hand sanitizers assessed had efficacy that meets World Health Organization (WHO) and PrEN12054 standards. From this study we are in agreement with the use of the locally made hand sanitizer
\end{abstract}


and Saraya. The two hand sanitizers had broad antibacterial spectrum. However, there is a need to evaluate efficacy and organoleptic properties using in vivo studies.

\section{Subject Areas}

Global Health

\section{Keywords}

Hand Sanitizer, Efficacy, Uganda, Antibacterial

\section{Background}

Health Care-Associated Infection (HCAI), also referred to as "nosocomial" or "hospital" acquired infection, is an infection occurring in a patient during the process of care in a hospital or other health care facility which was not present or incubating at the time of admission [1]. Hand transmission of infections is a common way of spreading nosocomial infections as hands are colonized permanently by physiological flora and temporarily depending on the precise nature of the health worker duties [2].

Hospital and Community acquired infections are escalating and pose a serious public health problem worldwide [3]. It is estimated that at any one time, more than 1.4 million people worldwide are suffering from infections acquired in hospitals [4].

A study by Agaba et al., (2017) revealed that the major isolated bacteria in nosocomial bacterial infections in Ugandan intensive care units were Klebsiella pneumoniae (30\%), Acinetobacter spp. 22\%, Staphylococcus aureus $14 \%$ and the MDR E. coli (50\%) [5].

The Centers for Disease Control and Prevention, the World Health Organization, and many other health experts promote hand hygiene as the single most important measure in the prevention of hospital-acquired infections. A study done by Reena Rajkumari (2015) showed the importance of proper hand hygiene in reducing the incidence of nosocomial infections. The use of hand sanitizers has become a cornerstone in clinical practice for the prevention of disease transmission between practitioners and patients [6].

Alcohol based hand sanitizers are recommended as a primary means for hand hygiene in healthcare settings when hands are not visibly soiled [1]. Traditionally many alcohol based hand sanitizers have relied on ethanol $(60 \%-70 \%)$ for bactericidal action [7].

Use of hand sanitizers has become a cornerstone in clinical practice for the prevention of disease transmission between practitioners and patients. There are a number of hand sanitizers sold on the Ugandan market with labels on their package claiming that the hand sanitizer can kill $99.999 \%$ of germs; however, no 
data locally is available to support this claim. In Jinja referral Hospital, there are two hand sanitizer brands (with some being locally made) whose efficacy remains unknown.

In 2017, a study by Ochwoto et al. in Kenya revealed that more than fifty percent $(50 \%)$ of the selected hand sanitizers in the Kenyan market had efficacy that falls below the World Health Organization (WHO) and DIN EN 1500:2013 standards [8].

This study aimed at evaluating the antibacterial efficacy of JRRH locally made Alcohol Based Hand Sanitizer (ABHS) and commonly available commercial hand sanitizer commonly used in healthcare facilities in Uganda. This was to address the concern about the burden of health care associated infections and the development of antibiotic resistant organisms with improved hand hygiene using effective Alcohol Based Hand Sanitizers.

\section{Methodology}

\subsection{Study Area}

Jinja Regional Referral Hospital (RRH) is located in southeastern Uganda, approximately 87 kilometers east of Kampala, Uganda. It is the largest hospital in eastern Uganda, with a bed capacity of 600 and serves as referral for Bugiri, Iganga, Jinja, Kaliro, Kamuli, Mayuge, Kayunga and parts of Mukono district. It has an infection prevention committee and locally made hand sanitizer in addition to commercial hand sanitizer for use by its staff. The hospital had a star 4 Laboratory that also served as a referral for 23 sites. The Laboratory was preparing for accreditation.

\subsection{Study Design}

This was an in in vitro laboratory-based experimental study. A suspension test for the demonstration of bactericidal activity of disinfectants in vitro (prEN12054) is used in Europe and EN 1500 for in vivo protocols [9].

\subsection{Study Population}

These were; Locally Made ABHS and Saraya Hand sanitizer, Purposive sampling method was applied. We tested two brands of hand sanitizers that were available at the hospital during the time of the study which were compared to a reference standard (60\% IPA).

\subsection{Materials and Methods}

The standard organisms that represent the commonly isolated pathogens causing nosocomial infections in Uganda were used in the experiment. These included; Klebsiella pneumoniae (ATCC 13883), Escherichia coli (ATCC 25922), and $S$. aureus (ATCC 25923). The culture media used in the study were blood agar and Muller Hinton Agar; they will be prepared using SOPS and the Microbiology laboratory at JRRH. Verified timers, autoclaves, water baths, biosafety 
cabinets, weighing scales and $\mathrm{pH}$ meters of the study site laboratory were used.

Data collection forms; Percentage bacterial growth reduction and Time-kill $\log$.

\subsection{Hand Sanitizers}

Two brands of alcohol-based hand sanitizers were found at Jinja Hospital, Saraya and locally made Alcohol based sanitizer.

$60 \%$ Isopropyl alcohol and neutralizer that contained Polysorbate80 were purchased from Joint medical stores, Kampala.

\subsubsection{Preparation of 0.5 McFarland Standard}

Mcfarland 0.5 turbidity standard was prepared according to the method recommended by the National Committee for Clinical Laboratory standards (NCCLS, 1999), the standard was prepared by adding $0.5 \mathrm{~mL}$ of $1.175 \% \mathrm{w} / \mathrm{v}$ Barium Chloride and $99.5 \mathrm{~mL}$ of $15 \% \mathrm{w} / \mathrm{v}$ Sulphuric acid. This was mixed well and then aliquots were made into test tubes identical to the ones used in preparing inoculum suspensions of the test organisms.

The accuracy of the density of the standard was verified using a spectrophotometer. The tubes were used 24 hours after preparation [10].

\subsubsection{Suspension Method}

In this study, the suspension method for the demonstration of bactericidal activity prEN12054, a European standard method for evaluating antibacterial efficacy of hand sanitizers was used.

Counts of the test organisms were reduced by factors exceeding $10^{5}$ fold within 30s for a hand sanitizer to be considered effective [11].

A sterile loop was used to pick a loopful of inoculum from a pure culture of the test organism.

This was then transferred and suspended in a tube of sterile normal saline, the tube was compared with the turbidity standard and the density of the organism was adjusted to that of the standard by adding more bacteria or more sterile saline [12].

A sample prepared from $0.5 \mathrm{McF}$ arland Standard containing a bacterial suspension of between $1.0 \times 10^{8}$ and $1.5 \times 10^{8} \mathrm{CFU} / \mathrm{ml}$ was the stock count of the different standard organisms, E. coli $1.5 \times 10^{8}, S$. aureus $1.45 \times 10^{8}$ and $K$. pneumoniae $1.5 \times 10^{8}$.

The test sample contained $900 \mu \mathrm{l}$ of sterile normal saline with $100 \mu \mathrm{l}$ of bacteria culture of the above concentrations to make bacteria suspension of $1 \mathrm{~mL}$ and from that, serial dilutions were made.

$1 \mathrm{~mL}$ of hand sanitizer was added to $1 \mathrm{~mL}$ of bacteria suspensions at different concentrations in different tubes and a timer was started whenever a hand sanitizer was being added to a bacteria suspension to stop the antibacterial activity at 15 seconds, 30 seconds and 45 seconds this was achieved by adding $1 \mathrm{~mL}$ of neutralizer that contained polysorbate 80 and physiological saline. The mix- 
tures were vortexed for 10 seconds and plated on Blood agar and Muller Hinton Agar.

The preparations were incubated at $37^{\circ} \mathrm{C}$ for 24 hours. Colony forming units were counted and the difference in the number of colony forming units between the baseline count and the test were recorded.

Baseline counts were the standardized preparations without hand sanitizer.

Positive control (Normal saline + Hand sanitizer) and negative controls (Normal saline + Bacteria + No Hand sanitizer) were included in the experiment.

The Percentage Growth Reduction factors (RF) were calculated using a formula;

$$
\% \text { Growth Reduction }=\frac{\text { Initial count }(\mathrm{CFU} / \mathrm{ml})-\text { Test count result }(\mathrm{CFU} / \mathrm{ml})}{\text { Initial } \operatorname{count}(\mathrm{CFU} / \mathrm{ml})} \times 100
$$

Log reduction factors were also calculated.

An increase in RF demonstrated an increase in the effectiveness or kill rate of bacterial growth.

\subsubsection{Effect of a Neutralizer Containing Polysorbate 80}

Effective neutralization of chemical biocides is the first step in the accurate evaluation of hand sanitizers to avoid over estimation of antibacterial activity. In this study we screened a neutralizer containing Polysorbate 80. Neutralizer efficacy was determined by comparing the recovery of the challenging index microorganisms from neutralizer with and without ABHS.

Acceptable neutralizer efficacy and neutralizer toxicity ratios are defined as $\geq 0.75$ (75\%) recovery [13].

Neutralizer toxicity was assessed against Klebsiella pneumoniae (ATCC 13883), Escherichia coli (ATCC 25922), and $S$. aureus (ATCC 25923) The PN-EN 13727 method used Standard bacterial suspension containing between $1.0 \times 10^{8}$ and $1.5 \times 10^{8} \mathrm{CFU} / \mathrm{ml}$. Bacterial suspensions of the three organisms were standardized to $10^{2} \mathrm{CFU}$. The effect of neutralizer toxicity on the microorganisms was tested in 10-seconds interactions of the neutralizer with bacteria suspension.

1 in 10 dilution was produced by adding $100 \mu \mathrm{l}$ of bacterial suspension to 900 $\mu \mathrm{l}$ of Neutralizer a second set of experiment was a control set that had 1:10 dilution of $100 \mu \mathrm{l}$ bacterial suspension and $900 \mu \mathrm{l}$ distilled water. The mixture was vortexed for 10s and plated on Mueller Hinton Agar, incubated at $37^{\circ} \mathrm{C}$ for 22 hours.

Viable colony forming units were counted on two sets of experiments and percentage bacteria recovery calculated.

\subsection{Data Quality Control}

All instruments used in the study were validated by competent qualified service providers. 
There was double entry of data into Microsoft excel software.

American Type Culture Collection (ATCC) Standard organisms were used and no expired products were used in the study.

Positive and negative control samples were included in the assays.

\subsection{Data Management and Analysis}

The obtained raw data were double entered into Microsoft Excel, Version 5.0 (Microsoft Redmond, WA, USA) and then graphs plotted. Data were exported to graph pad prism software to plot a time to kill curve.

Log and Percent reduction for the standard microorganisms were calculated and presented using tables.

\subsection{Ethical Considerations}

The study protocol was approved by Mbarara University of Science and Technology, Department of Medical Laboratory Science (Reference: MUST/MLS/031) and Jinja Regional Referral Hospital Research Ethics Committee (REC) granted Permission/ethical waiver where the study was conducted.

\subsection{Limitations}

The study was limited to bacteria; no viruses and yeasts were handled because of capacity to isolate viruses and a method EN1275 is used for testing efficacy against yeasts and viruses.

The study was in vitro and did not closely stimulate real world conditions of Hand Sanitizer use.

\subsection{Research Dissemination Plan}

Results from this study were shared with Jinja Regional Referral Hospital (JRRH) Administration and Infection Control and Prevention Committee (IPC) and also compiled into a dissertation, a copy of which was submitted to the $\mathrm{Li}$ brary in the Department of Medical Laboratory Science of Mbarara University of Science and Technology Abstracts of this work have been sent and accepted at Colorado WASH symposium and ICREID conference.

\section{Results}

Hand sanitizers, standard and Neutralizer used in the experiment and their composition as shown in Table 1.

\subsection{Efficacy of JRRH Locally Made Alcohol Based Hand Sanitizer (ABHS)}

The JRRH locally made ABHS was effective against all the test organisms. The antibacterial effectiveness was assessed by counting Colony Forming Units (CFU) after treatment with the hand sanitizer for 15 seconds contact time. Logarithmic reduction factors and percentage growth reductions calculated as shown 
Table 1. Hand sanitizers used in the experiment and their compositions.

\begin{tabular}{cll}
\hline No. & \multicolumn{1}{c}{ Product } & \multicolumn{1}{c}{ Composition/Ingredient } \\
\hline 1 & JRRH Locally Made ABHS & $\begin{array}{l}\text { 80\% Ethanol, Hydrogen peroxide, Deionized } \\
\text { water and Glycerin }\end{array}$ \\
& & $\begin{array}{l}70 \% \text { Ethanol, water, Glycerin, Isopropyl } \\
\text { Myristate, Allantoin and Phosphoric acid }\end{array}$ \\
2 & Saraya & $99 \%$ Isopropyl alcohol, Water \\
3 & 60\% Isopropyl alcohol & 1\% Physiological peptone, Tween 80
\end{tabular}

in Table 2. The highest reduction factors were recorded against $E$. coli and $K$. pneumoniae at 7.18 and $S$. aureus at 7.16 whose baseline counts were $1.5 \times 10^{7}$, $1.5 \times 10^{7}$ and $1.45 \times 10^{7} \mathrm{CFU}$ respectively (Table 2 ).

\subsection{Efficacy of Commonly Available Commercial Hand Sanitizer (Saraya)}

Saraya hand sanitizer was effective against all the test organisms. The antibacterial effectiveness was assessed by counting colony forming units (CFU) after treatment with the hand sanitizer for 15 seconds contact time. Logarithmic reduction factors and percentage growth reductions calculated as shown in Table 3. The highest reduction factors were recorded against $E$. coli and $K$. pneumoniae at 7.18 and $S$. aureus at 7.16 whose baseline counts were $1.5 \times 10^{7}$, $1.5 \times 10^{7}$ and $1.45 \times 10^{7} \mathrm{CFU}$ respectively and post counts after treatment with sanitizer were $1 \mathrm{CFU}$ across all organisms with percentage growth reduction of 99.99999\%.

\subsection{Efficacy of $60 \%$ Isopropyl Alcohol (Standard)}

The controls hand sanitizer (60\% IPA) was satisfactory for suspension test for all the test organisms used in the studyin agreement with prEN 12054 method (Table 4).

\subsection{Comparison of the Antibacterial Efficacy of Saraya and Locally Made Hand Sanitizer}

Both Locally made Alcohol Hand sanitizer and Saraya had a bacterial percentage reduction of $99.99999 \%$ at 15 and 30 seconds contact time (Table 5 and Table 6) against E. coli, $K$. pneumonia and $S$. aureus whose baseline counts were $1.5 \times$ $10^{7}, 1.5 \times 10^{7}$ and $1.45 \times 10^{7} \mathrm{CFU}$ respectively whose post counts after treatment with sanitizers were $1 \mathrm{CFU}$ across all organisms.

\subsection{Bacterial Growth Reduction Values for Different Hand Sanitizers}

The highest reduction factors were recorded against $E$. coli and $K$. pneumoniae at 7.18 each and $S$. aureus at 7.16 whose baseline counts were $1.5 \times 10^{7}, 1.5 \times 10^{7}$ and $1.45 \times 10^{7}$ colony forming units respectively (Figure 1 ). 
Table 2. $\log _{10}$ reduction and percentage growth reduction values of JRRH locally made alcohol hand sanitizer (L.ABHS) at 15 seconds.

\begin{tabular}{ccc}
\hline Organism & Log Reduction Factor & Percentage Growth Reduction \\
\hline Escherichia coli & 7.18 & $99.99999 \%$ \\
Staphylococcus aureus & 7.16 & $99.99999 \%$ \\
Klebsiella pneumoniae & 7.18 & $99.99999 \%$ \\
\hline
\end{tabular}

Table 3. $\log _{10}$ and percentage growth reduction values of saraya hand sanitizer.

\begin{tabular}{ccc}
\hline Organism & Log Reduction Factor & Percentage Growth Reduction \\
\hline E. coli & 7.18 & $99.99999 \%$ \\
S. aureus & 7.16 & $99.99999 \%$ \\
K. pneumoniae & 7.18 & $99.99999 \%$ \\
\hline
\end{tabular}

Table 4. $\log _{10}$ and percentage growth reduction values of $60 \%$ isopropyl alcohol.

\begin{tabular}{ccc}
\hline Organism & Log Reduction Factor & Percentage Growth Reduction \\
\hline E. coli & 7.18 & $99.99999 \%$ \\
S. aureus & 7.16 & $99.99999 \%$ \\
K. pneumoniae & 7.18 & $99.99999 \%$ \\
\hline
\end{tabular}

Table 5. Percentage bacterial growth reduction at 15 seconds of contact time with different hand sanitizers.

\begin{tabular}{cccc}
\hline \multirow{2}{*}{ Hand sanitizer } & \multicolumn{3}{c}{ Percentage Growth Reduction } \\
\cline { 2 - 4 } & E. coli & S. aureus & K. pneumoniae \\
\hline JRRH Locally Made ABHS & $99.99999 \%$ & $99.99999 \%$ & $99.99999 \%$ \\
Saraya & $99.99999 \%$ & $99.99999 \%$ & $99.99999 \%$ \\
60\% Isopropyl Alcohol & $99.99999 \%$ & $99.99999 \%$ & $99.99999 \%$ \\
\hline
\end{tabular}

Table 6. Percentage bacterial growth reduction at 30 seconds of contact time with different hand sanitizers.

\begin{tabular}{cccc}
\hline \multirow{2}{*}{ Hand sanitizer } & \multicolumn{3}{c}{ Percentage Growth Reduction } \\
\cline { 2 - 4 } & E. coli & S. aureus & K. pneumoniae \\
\hline JRRH Locally Made ABHS & $99.99999 \%$ & $99.99999 \%$ & $99.99999 \%$ \\
Saraya & $99.99999 \%$ & $99.99999 \%$ & $99.99999 \%$ \\
60\% Isopropyl Alcohol & $99.99999 \%$ & $99.99999 \%$ & $99.99999 \%$ \\
\hline
\end{tabular}

\subsection{Bacteria Kill-Time Curve against Different Hand Sanitizers}

Both sanitizers reduced the baseline counts from $1.5 \times 10^{7} \mathrm{CFU}$ to $1 \mathrm{CFU}$ within 15 seconds of contact time, post exposure counts remained constant (1 CFU) at 30 and 45 seconds contact time (Figure 2). 


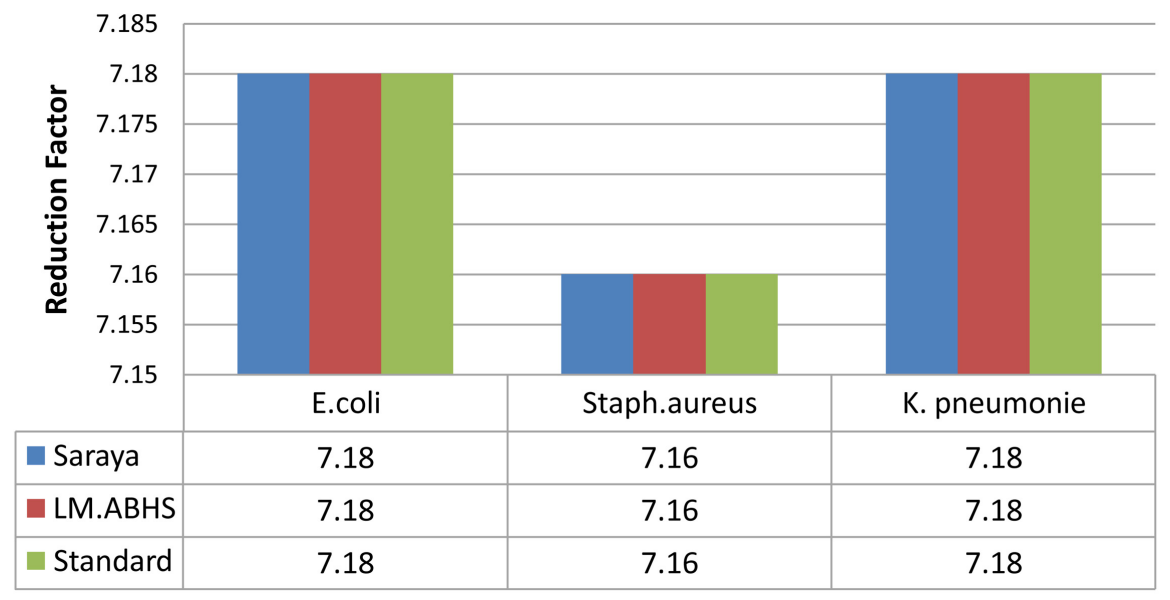

Figure 1. Bacterial growth reduction values for different hand sanitizers.

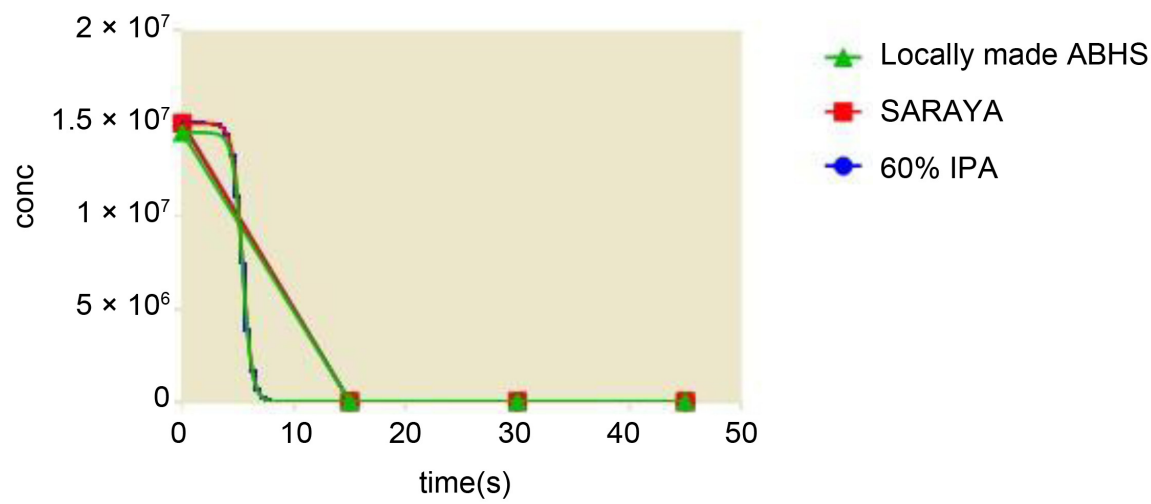

Figure 2. Bacteria kill-time curve against different hand sanitizers.

\subsection{The Effect of Neutralizer Toxicity on the Microorganisms}

The neutralizer used in the study was found not to be toxic to the microorganisms used; more than $80 \%$ of the organisms were recovered after being treated with the neutralizer for 10 seconds contact time (Table 7).

\section{Discussion, Conclusion and Recommendation}

\subsection{Discussion}

Both Saraya and JRRH Locally made ABHS mentioned ethanol as their active ingredient. Efficacy was independent of the ethanol concentration in each brand (Saraya $70 \%$ and locally made $80 \%$ alcohol). In this study alcohol concentrations were not adjusted, sanitizers were used in their original form from the manufacturer/producers. Results of this study are similar to Sax et al., (2009) where Alcohol based products typically contained between $60 \%$ and $95 \%$ alcohol, in form of ethanol or Isopropanol. At those concentrations, alcohol immediately denatured proteins effectively neutralizing certain types of organisms [14].

In agreement with prEN 12054, locally made ABHS and Saraya were found to exceed a $10^{5}$-fold reduction of all the three ATCC strains (Klebsiella pneumoniae (ATCC 13883), Escherichia coli (ATCC 25922), and S. aureus (ATCC 25923) 
Table 7. The effect of Neutralizer toxicity on the microorganisms tested in $10 \pm 2$ seconds interaction.

\begin{tabular}{ccccc}
\hline Test Organism & $\begin{array}{c}\text { Bacterial } \\
\text { Suspension }\end{array}$ & $\begin{array}{c}\text { Validation } \\
\text { Suspension }\end{array}$ & $\begin{array}{c}\text { Suspension with } \\
\text { Neutralizer }\end{array}$ & $\begin{array}{c}\text { Recovery } \\
\text { Bacteria } \\
\text { Percentage }\end{array}$ \\
\hline S. aureus & $10^{2}$ & $110 \pm 3 \mathrm{Cfu} / \mathrm{ml}$ & $100 \pm 5 \mathrm{Cfu} / \mathrm{ml}$ & $90.9 \%$ \\
E. coli & $10^{2}$ & $105 \pm 6 \mathrm{Cfu} / \mathrm{ml}$ & $100 \pm 6 \mathrm{Cfu} / \mathrm{ml}$ & $95.2 \%$ \\
K. pneumoniae & $10^{2}$ & $108 \pm 5 \mathrm{Cfu} / \mathrm{ml}$ & $90 \pm 6 \mathrm{Cfu} / \mathrm{ml}$ & $83.3 \%$ \\
\hline
\end{tabular}

within $15 \mathrm{~s}$ (Table 1 and Table 2). This study is in compliance with WHO (2009) that describes an efficacious locally produced hand sanitizer that reduced microorganism by a $10^{5}$-fold reduction and above [9].

According to the label on Saraya hand sanitizer that shows the hand sanitizer can kill $99.999 \%$ of bacteria, bacterial percentage reductions were calculated and this study is in agreement with the label however there was no data on efficacy of the locally produced hand sanitizer nevertheless this study found out that the sanitizer can reduce bacteria by $99.99999 \%$ (Table 4 and Table 5).

The two hand sanitizers have a broad spectrum antimicrobial activity. A study by Vardhaman showed that alcohol based hand sanitizers were effective against Staphylococcus aureus, Staphylococcus epidermidis, Pseudomonas aeruginosa, Escherichia coli and Enterococcus faecalis [15].

The Positive control (60\% Isopropyl alcohol) had a percentage growth reduction of $99.999 \%$, while the negative controls (Neutralizer) had no effect on the growth reduction hence these were satisfactory for suspension test for all the test organisms used in the study.

This study showed that the use of a neutralizer that contained Polysorbate 80 (Tween 80 ) was non-toxic on both gram negative and gram positive bacteria since more than $80 \%$ of the bacteria were recovered after being treated with the neutralizer for 10 seconds contact time. Acceptable neutralizer efficacy and neutralizer toxicity ratios are defined as $\geq 0.75$ (75\%) recovery [13].

Adams et al. (2005) reported that mixture of $0.2 \%$ Tween $80,1.17 \%$ Lecithin, $0.1 \%$ triton in distilled water is an effective neutralizer for Alcohol hand gel with whom we are in agreement according to our results. Another study by Norhan Sheraba et al. (2012) shows that Tween 80 in 1\% physiological peptone was the most effective neutralizer contrary, Mohamed (2004) found Letheen broth to be an effective neutralizer [16].

\subsection{Conclusions}

The hand sanitizers used had 100\% efficacy that meets World Health Organization (WHO) standards that require $5 \log _{10} \mathrm{CFU} / \mathrm{mL}$ Reduction of bacteria within 30 seconds of contact time. From this study we recommend the use of the JRRH locally made hand sanitizer and Saraya. Hand rubbing time can be reduced from $30 \mathrm{~s}$ to $15 \mathrm{~s}$. 
Local production of ABHS should be adopted in tertiary facilities as the preferred means of providing for ABHS for their staff at the "point of care" (POC). This is recommended by WHO and satisfies the urgent need to make available hand sanitizers in healthcare facilities at the POC to help in reducing transmittable infections [1].

\section{Recommendations}

With these study findings, we recommend that future studies to;

1) Include other micro-organisms such as known resistant bacteria, clinical isolates, viruses and fungi.

2) Evaluate the efficacy and organoleptic properties of hand sanitizer using in-vivo studies that stimulate real world of hand sanitizer usage.

3) Evaluate more neutralizers unlike only one used in this study for efficacy and toxicity.

\section{Acknowledgements}

We acknowledge Mbarara University Department of Medical Laboratory Sciences, Uganda Institute of Allied Health and management sciences-Mulago, Jinja Regional Referral Hospital management and Mr. Olum the Principal Pharmacist JRRH for the work done toward seeing this project a success. We would like to also acknowledge Wewedru Izaale for reviewing our work.

\section{Conflicts of Interest}

Researchers had no competing interests.

\section{Availability of Data and Materials}

Data and materials will be availed upon written request.

\section{Funding}

No external funding was received

\section{Authors' Contributions}

All authors made substantial contribution to protocol development provided monetary contribution, instrument development, data collection and analysis, LA supervised and gave technical support to the entire project.

\section{References}

[1] WHO (2009) Guidelines on Hand Hygiene in Health Care. World Health Organization, Geneva.

[2] Allegranzi, B. (2009) Role of Hand Hygiene in Health Care Associated Infection Prevention. Journal of Hospital Infection, 73, 305-315. https://doi.org/10.1016/j.jhin.2009.04.019

[3] Hassan, A.O., Muhibi, M.A. and Adebimpe, W.O. (2012) A Survey of Enterobacte- 
riaceae in Hospital and Community Acquired Infections among Adults in a Tertiary Health Institution in Southwestern Nigeria. African Journal of Microbiology Research, 6, 5162-5167.

[4] Madan, K. and Thakral, S. (2012) Comparative Evaluation of Efficacy of Alcoholic vs. Non-Alcoholic Hand Sanitizers. International Journal of Life Science and Pharma Research.

[5] Agaba, P., Tumukunde, J., Tindimwebwa, J.V.B. and Kwizera, A. (2017) Nosocomial Bacterial Infections and Their Antimicrobial Susceptibility Patterns among Patients in Ugandan Intensive Care Units: A Cross Sectional Study. BMC Research Notes, 10, 349. https://doi.org/10.1186/s13104-017-2695-5

[6] Rajkumari, R. (2015) Evaluation of the Efficacy of Six Different Hand Sanitizers Commonly Available on the Indian Market. International Journal of Pharma and Bio Sciences, 6, B984-B991.

[7] Bondurant, S.W., Duley, C.M. and Harbell, J.W. (2019) Demonstrating the Persistent Antibacterial Efficacy of a Hand Sanitizer Containing Benzalkonium Chloride on Human Skin at 1, 2, and 4 Hours after Application. American Journal of Infection Control, 47, 928-932. https://doi.org/10.1016/j.ajic.2019.01.004

[8] Ochwoto, M., Muita, L., Talaam, K., Wanjala, C., Ogeto, F., Wachira, F., et al. (2017) Anti-Bacterial Efficacy of Alcoholic Hand Rubs in the Kenyan Market, 2015. Antimicrobial Resistance \& Infection Control, 6, 17. https://doi.org/10.1186/s13756-017-0174-3

[9] Kampf, G., Meyer, B. and Goroncy-Bermes, P. (2003) Comparison of Two Test Methods for the Determination of Sufficient Antimicrobial Efficacy of Three Different Alcohol-Based Hand Rubs for Hygienic Hand Disinfection. Journal of Hospital Infection, 55, 220-225. https://doi.org/10.1016/S0195-6701(03)00255-X

[10] Kiehlbauch, J.A., Hannett, G.E., Salfinger, M., Archinal, W., Monserrat, C. and Carlyn, C. (2000) Use of the National Committee for Clinical Laboratory Standards Guidelines for Disk Diffusion Susceptibility Testing in New York State Laboratories. Journal of Clinical Microbiology, 38, 3341-3348. https://doi.org/10.1128/JCM.38.9.3341-3348.2000

[11] Rotter, M. (2004) European Norms in Hand Hygiene. Journal of Hospital Infection, 56, 6-9. https://doi.org/10.1016/j.jhin.2003.12.024

[12] Vandepitte, J., Engbaek, K., Rohner, P., Piot, P. and Heuck, C.C. (2003) Basic Laboratory Procedures in Clinical Bacteriology. 2nd Edition, World Health Organization, Geneva.

[13] Sutton, S.V., Proud, D.W., Rachui, S. and Brannan, D.K. (2002) Validation of Microbial Recovery from Disinfectants. Journal of Pharmaceutical Science and Technology, 56, 255-266.

[14] Sax, H., Allegranzi, B., Chraiti, M.-N., Boyce, J., Larson, E. and Pittet, D. (2009) The World Health Organization Hand Hygiene Observation Method. American Journal of Infection Control, 37, 827-834. https://doi.org/10.1016/j.ajic.2009.07.003

[15] Jain, V.M., Karibasappa, G.N., Dodamani, A.S., Prashanth, V.K. and Mali, G.V. (2016) Comparative Assessment of Antimicrobial Efficacy of Different Hand Sanitizers: An in Vitro Study. Dental Research Journal, 13, 424-431. https://doi.org/10.4103/1735-3327.192283

[16] Sherabah, N., Yassin, A., Mohamed, A. and Amin, M. (2012) Efficacy and Toxicity of Neutralizers against Disinfectants and Antiseptics Used in Vaccine Production Facility. African Journal of Microbiology Research, 6, 6565-6571.

https://doi.org/10.5897/AJMR12.934 


\section{List of Abbreviations}

ABHR: Alcohol Based Hand Rub, ABHS: Alcohol Based Hand Sanitizer, ASTM: American Society for Testing Materials, E. coli: Escherichia coli, EN: European Norm, H.H: Hand Hygiene, HAI: Hospital Acquired Infection, HAI's: Hospital Acquired Infections, IPC: Infection Prevention and Control, $K$. pneumoniae: Klebsiella pneumoniae, MDR: Multi Drug Resistant, S. aureus. Staphylococcus aureus, SOPS: Standard Operating Procedures, Std: Standard. 